\title{
SUB-REGISTRO DE NASCIMENTO NO DISTRITO DE SÃO PAULO (1)
}

\begin{abstract}
Foram coletados os dados sôbre os nascimentos ocorridos em 1965, o fato de terem ou não sido registrados e qual o motivo, em caso negativo, por ocasião da investigacão sộbre «Reprodução Humana no Distrito de São Paulo», realizada em uma amostra da população de mulheres não solteiras, no grupo de 15 a 49 anos. Quando se considerou o prazo de inscrição de um nascimento até 15 dias, que é o prazo dado ao pai da crianca, o sub-registro foi de $4,5 \%$ e quando se considerou o prazo de 45 dias dilatado para quando a mãe inscreve o nascimento, o sub-registro foi de $3,2 \%$. O fator mais mencionado para o não-registro foi o relacionado com a responsabilidade do marido e em cêrca de $80 \%$ dos casos, o parto havia sido hospitalar.
\end{abstract}

\section{N T RODU C AO}

Um registro de nascimento completo é de suma importância para o planejamento e desenvolvimento de programas de saúde. Como por exemplo, a estimativa do número de imunizaçóes contra doenças infecciosas, para o cálculo dos reais coeficientes de mortalidade infantil, natalidade, mortalidade materna e demais coeficientes que envolvem a utilização do número de nascidos vivos, para estimativas intercensuais de população, construção de tábuas de sobrevivência, etc.

Para o Brasil, fora alguns estudos isolados (Moraes ${ }^{6}, 1949$; Rosado $^{\circ}, 1949$; SAAde ${ }^{10}, 1947$ e Scorzelli ${ }^{11}, 1947$ ) é bastante escassa a informação sôbre a eficácia do registro de nascimentos.

A Comissão do Censo das Américas (COTA) já recomendava que se realizasse por ocasiáo do censo de 1950, um teste sôbre a eficácia do registro de nascimentos (MORRISON \& BLEN ${ }^{7}, 1949$ ),
- que foi feito pelos Estados Unidos da América e alguns países da América Latina, entre êles, o Chile (CABELlo ${ }^{2}$, 1956). Para os Estados Unidos aquela prova sôbre a eficácia do registro de nascimentos foi a segunda de âmbito nacional (DunN *, 1941 e Shapiro \& Schachter ${ }^{15}$, 1952) e o Paraguai havia já realizado uma prova em 1944 (Ocampos \& BennetT $\left.{ }^{8}, 1946\right)$.

Também o primeiro Seminário Interamericano de Registro Civil ${ }^{12}$ (1955), no qual o Brasil não se fêz representar, sugeriu que as autoridades nacionais efetuassem estudos especiais para determinar a percentagem de omissão em cada um dos grupos principais de população, usando para isso os métodos mais adequados, segundo as circunstâncias. Entretanto, por ocasião do segundo SEMINÁRIo INTERAMERICANo de Registro GiviL ${ }^{13}$ (1964), quase a metade dos países participantes e que foram 25, reconheceu

(1) Da Cadeira de Estatistica Aplicada à Saúde Pública da Faculdade de Higiene e Saúde Pública da USP. 
MILANESI, M. L. \& SILVA, E. P. de C. - Sub-registro de nascimento no Dístrito de Sāo Paulo. Rev. Saúde públ., $2(1): 23-28$, jun. 1968.

que não se fêz nenhum estudo sôbre a matéria, alegando falta de experiência e escassez de recursos econômicos. Entre êles incluia-se o Brasil. Este segundo Seminário fêz novas recomendaçóes para que se fizessem estudos destinados a medir o grau de omissão e exatidão da informação, aproveitando, se possível, o censo de 1970, apresentando uma lista de doze métodos possíveis para êste tipo de estudo.

O método convencional para testar a eficiência do registro de nascimentos envolve a estimativa da população infantil, partindo das estatísticas de nascimentos e óbitos e a comparação desta com dados do censo. Tal método pressupōe que a população acusada pelo censo é a verdadeira, que o registro de óbitos é completo e que não existe migração da população infantil na área em consideração.

O método utilizado por ocasião do censo de 1950 nos Estados Unidos consiste em que durante a realização do censo, os recenseadores preenchem um pequeno formulário especial sôbre crianças que nasceram durante um período pré-fixado (3.4 meses) e que estão vivas por ocasiáo do recenseamento. Este formulário especial será confrontado com as inscrições de nascimentos obtidas em cartórios, bem como com os atestados de óbito de crianças nascidas no mesmo período. Tal método permite que se determine não só o sub-registro de nascimento, mas também a sub-enumeração censual da população infantil e possibilita a correção quantitativa do número de registros de nascimentos, uma vez que tais nascimentos passam a ser conhecidos.

Outro método indireto proposto por SwEE-Rock ${ }^{17}$ (1964), envolve a utilização de dois recenseamentos, da distribuição de mulheres por grupos qüinqüenais e filhos tidos, bem como leva em consideração a mortalidade materna entre os dois recenseamentos e, neste caso, o sub-registro é estabelecjdo para todo o período entre os dois censos.

\section{MATERIAL E METODOS}

Por ocasião da investigação sôbre Reprodução Humana no Distrito de São Paulo (Berquó ${ }^{1}, 1967$ ), surgiu a oportunidade de quantificar o sub-registro de nascimentos no distrito, perguntando-se àquelas mulheres que haviam tido um nascido vivo em 1965, se já haviam ou não registrado a criança e qual o motivo, em caso negativo.

Acreditamos que tal método de avaliação do sub-registro nos dará a indicação de um valor minimo para sua deficiência, uma vez que a tendência da entrevistada deva ser no sentido de ocultar o fato de não haver ainda registrado o filho e também pelo fato de que como não foi perguntado à mãe com quantos dias registrou a criança, é possível que das crianças declaradas como registradas, algumas o houvessem sido fora do prazo.

Para o estabelecimento do sub-registro, levou-se em consideração dois fatos contidos na lei e que se referem ao prazo legal para inscrição de um nascimento - 15 dias para o pai e 45 dias para a mãe. Foram, pois, feitas duas estimativas de sub-registro, isto é, o sub-registro levando-se em conta o prazo de 15 dias para inscrição $\left(R_{1}\right)$ e o sub-registro levando-se em conta o prazo de 45 dias para inscrição $\left(R_{2}\right)$.

Tendo aquela pesquisa sôbre Reprodução Humana no Distrito de São Paulo, baseado-se numa amostra de domicílios, estratificada com partilha proporcional, na qual os estratos foram os sub-distritos do Distrito de São Paulo (Silva ${ }^{16}$, 1968), empregou-se para aquelas estimativas, o estimador tipo índice combinado (Cochran ${ }^{3}$, 1963), cujas fórmulas inclusive as da respectiva variância, passamos a apresentar, após a indicação da notação empregada. 
MILANESI, M. L. \& SILVA, E. P. de C. - Sub-registro de nascimento no Distrito de Såo Paulo. Rev. Saúde públ., 2(1):23-28, jun. 1968.

Notação:

$\mathrm{X}=$ total de crianças nascidas vivas em 1965, que atingiram mais de 15 dias (mais de 45 dias) de vida;

$\mathrm{Y}=$ total de crianças nascidas vivas em 1965 , que atingiram mais de 15 dias (mais de 45 dias) de vida e que até 15 dias (até 45 dias) após o nascimento não foram registradas;

$\mathrm{R}=\frac{\mathrm{X}}{\mathrm{Y}}$ índice de sub-registro de nascimento;

$\hat{R}_{c}=\frac{\sum_{h}^{L} y_{h}}{\sum_{h}^{L} x_{11}}$ estimador por índice combinado do índice populacional $R$;

$\mathbf{x}_{\mathrm{h}}=$ total de crianças nascidas vivas de mulheres da amostra do estrato $h$, que na data de entrevista tinham mais de 15 dias (mais de 45 dias) de vida;

$\mathrm{y}_{\mathrm{h}}=$ total de crianças nascidas vivas de mulheres da amostra do estrato $h$, que na data da entrevista tinham mais de 15 dias (mais de 45 dias) de vida e que não haviam sido registradas;

$\mathbf{L}=$ número de estratos.

Variância de $\stackrel{\Lambda}{R}_{c}$

$$
\underset{\substack{\mathrm{R}_{\mathrm{c}} \\ \sigma^{2}}}{\mathrm{X}^{2}} \frac{\mathrm{N}-\mathrm{n}}{\mathrm{n}} \quad \Sigma \mathrm{N}_{\mathrm{h}}\left(\mathrm{S}_{\mathrm{yh}}^{2}+\mathrm{R}^{2} \mathrm{~S}_{\mathrm{xh}}^{2}-2 \mathrm{RS}_{\mathrm{yxh}}\right)
$$

$\mathrm{S}_{\mathrm{yh}}^{2}=\frac{\sum_{1}^{N_{\mathrm{h}}}\left(\mathrm{y}_{\mathrm{h} 1}-\overline{\mathrm{Y}}_{\mathrm{h}}\right)^{2}}{\mathrm{~N}_{\mathrm{h}}-1}$ quadrado médio da variável $y$ no estrato $h$

$\mathrm{S}_{\mathrm{xh}}^{2}=\frac{\sum_{1}^{\mathrm{N}_{\mathrm{h}}}\left(\mathrm{x}_{\mathrm{hi}}-\overline{\mathrm{X}}_{\mathrm{h}}\right)^{2}}{\mathrm{~N}_{\mathrm{h}}-1}$ quadrado médio da variável $x$ no estrato $h$

$S_{y \times h}=\frac{\sum_{i}^{N_{h}}\left(y_{h i}-\bar{Y}_{h}\right)\left(x_{h i}-\bar{X}_{h}\right)}{N_{h}-1} \quad \begin{gathered}\text { covariância entre as variáveis } \\ y \text { e } x \text { no estrato } h\end{gathered}$ 
MILANESI, M. L. \& SILVA, E. P. de C. - Sub-registro de nascimento no Distrito de São Paulo. Rev. Saúde públ., 2(1):23-28, jun. 1968.

$\mathrm{N}_{\mathrm{b}}=$ número de unidades domiciliárias no estrato $h^{(1)}$

$$
\mathrm{N}=\underset{\mathrm{h}}{\mathrm{L}} \mathrm{N}_{\mathrm{h}}
$$

$\mathrm{x}_{\mathrm{hi}}=$ número de nascidos vivos em 1965, da mulher residente no domicílio $i$ do estrato $h$, que atingiram mais de 15 dias (mais de 45 dias) de vida;

$\mathrm{y}_{\mathrm{hi}}=$ número de nascidos vivos, dentre os $x_{h i}$ citados antes e que até 15 dias (até 45 dias) após o nascimento não foram registrados.

Estimador de $\sigma^{2}{ }_{R_{\mathrm{c}}}^{A}$

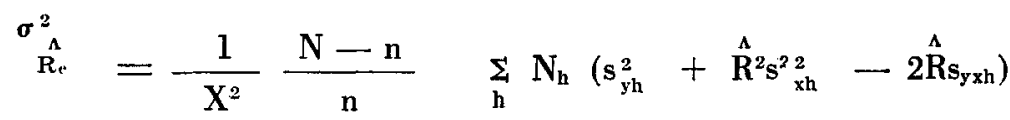

onde

$$
\begin{aligned}
& \mathbf{s}_{\mathrm{yh}}^{2}=\frac{\sum_{j}^{n_{h}}\left(y_{h j}-\overline{y_{h}}\right)^{2}}{n_{h}-1} \\
& s_{x h}^{2}=\frac{\sum_{j}^{n_{h}}\left(x_{h j}-\overline{x_{h}}\right)^{2}}{n_{h}-1} \\
& s_{y x h}=\frac{\sum_{j}^{n_{h}}\left(y_{h j}-\overline{y_{h}}\right)\left(x_{h j}-\bar{x}_{h}\right)}{n_{h}-1}
\end{aligned}
$$

$x_{\mathrm{hj}}=$ número de nascidos vivos em 1965 da mulher $j$ da amostra do estrato $h$, e que na data da entrevista tinham mais de 15 dias (mais de 45 dias);

$\mathrm{y}_{\mathrm{hj}}=$ número de nascidos vivos dentre os $x_{h j}$ citados antes e que na data da entrevista ainda não haviam sido registrados.

$$
\begin{aligned}
& \bar{x}_{h}=\frac{x_{h}}{n_{h}}=\frac{\sum_{j}^{n_{h}} x_{h j}}{n_{h}} \\
& \bar{y}_{h}=\frac{y_{h}}{n_{h}}=\frac{\sum_{j}^{n_{h}} y_{h j}}{n_{h}}
\end{aligned}
$$

(1) Os tamanhos dos estratos $\mathrm{N}_{\mathrm{h}}$ foram estimados com base nos dados do censo éscolar e na estimativa do total de mulheres não-solteiras em 1965, no Distrito de São Paulo. 
MILANESI, M. L. \& SILVA, E. P. de C. - Sub-registro de nascimento no Distrito de são Paulo. Rev. Saúde públ., 2(1):23-28, jun. 1968.

\section{RESULTADOS E DISCUSSAO}

Houve um total de 366 nascidos vivos entre as 3.009 mulheres que entraram na amostra. Dêstes, 10 não tinham ainda 15 dias de vida por ocasião da entrevista e 50 não haviam ainda completado 45 dias de vida. Daqueles que já haviam completado 15 dias de vida, deixaram de ser registradas, até esta época, 16 crianças. Daqueles que já haviam completado 45 dias, 10 deixaram de ser registradas até aquela época.

Aplicando-se o estimador mencionado e calculando-se o respectivo êrro padrão, chegamos aos seguintes resultados:

1 - $O$ indice de sub-distrito de nascimentos quando se levou em consideração o prazo de 15 dias para a inscrição foi

$$
\stackrel{\mathrm{R}}{\mathrm{R}}_{1}=4,5 \%
$$

O êrro padrão para esta estimativa foi $\sigma\left(R_{1}\right)=1,09 \%$, o que significa que depositamos 95\% de confiança que o intervalo $2,4 \%$ - $6,6 \%$ contenha o verdadeiro valor do índice mínimo de sub-registro de nascimentos.

2 - $O$ indice de sub-registro de nascimentos quando se levou em consideração o prazo de 45 dias para inscrição foi

$$
\stackrel{\mathbf{R}}{2}_{2}=3,2 \%
$$

$O$ êrro padrão para esta estimativa foi $\mathbf{\sigma}_{\mathbf{R}}=0,98 \%$, o que significa que depositamos $95 \%$ de confiança que o intervalo $1,2 \%-5,1 \%$ contenha o verdadeiro valor do indíce mínimo de sub-registro de nascimentos.

Estimativas de sub-registro de nascimentos feitas pelo $\operatorname{IBGE}^{5}$ (1964), revelaram que para o Brasil, como um todo, $50.86 \%$ dos nascimentos ocorridos em 1960 foram registrados no mesmo ano e, quando se levou em consideração o número de registrados em 1960, nascidos em 1960 ou em anos anteriores sôbre o total de nascidos vivos em 1960, o índice de registrados eleva-se a $81,44 \%$. Tais índices variaram, naturalmente, de um Estado da Federação para outro. Para o Estado de São Paulo, o sub-registro estimado naquele trabalho, foi de 9,73\% (nascidos e registrados no mesmo ano), o que é coerente com os nossos resultados, uma vez que se espera que o sub-registro para o Estado como um todo, seja maior do que para o Distrito da Capital.

Um outro método possivel para detectar o índice de sub-registro teria sido comparar o total de nascidos vivos registrados, dado pelo registro civil com o total de nascimentos estimados através da amostra. Para tal estimativa, só poderíamos calcular o índice de sub-registro para o prazo legal total, estipulado por lei. Empregando tal método, obteve-se como estimativa do total de nascimentos no distrito, 135.785 e as estatís. ticas oficiais deram 131.129 nascidos vivos, registrados em 1965. Isso significa que por êste processo o sub-registro de nascimentos foi da ordem de $3,4 \%$, valor êste bastante próximo do obtido através do primeiro processo empregado, isto é, $\stackrel{\mathrm{R}}{\mathrm{*} 2}_{2}=3,2 \%$.

Entre os motivos citados para o não registro, foram mencionados entre os 16 casos não registrados até 45 dias:

1) fator relacionado a problemas de responsabilidade do marido.. .7 casos

2) problema monetário 3 casos

3) fator de tempo ...... 2 casos

4) problema de ilegitimidade 2 casos

5) outros

2 casos

E interessante notar que dos 16 casos, 13 dêles foram partos hospitalares, o que vem confirmar um dos fatos fundamentais, considerados no segundo SEMINÁRIo Interamericano DE Registro CiviL ${ }^{\text {t4 }}$ (1965) para o melhoramento do Registro Civil, isto é, a necessidade do estabelecido de oficinas filiais de registro em hospitais, clínicas e maternidades e de uma campanha junto à direção de tais estabelecimentos, no sentido de mostrar a importância do papel que desempe- 
MILANESI, M. L. \& SILVA, E. P. de C. - Sub-registro de nascimento no Distrito de São Paulo. Rev. Saúde públ., 2(1):23-28, jun. 1968.

nham na declaração oportuna e adequada dos fatos vitais, como nascimentos, óbitos, óbitos fetais.

\section{S U M M A R Y}

Data have been collected on the births occurred in 1965, on whether they have been registered and on the reason in case they have not, at the time of the investigation on: Human Reproduction in the District of São Paulo carried out with basis on a sample of the non-single, 15 to 49 years old female population.

When the time considered for registration of a birth was up to 15 days, which is the case when the father is the one who registers the child, the underregistration was $4.5 \%$, but with the extended time of 45 days, when the mother is supposed to register the birth, the under-registration was $3.2 \%$. The main factor for not registering the births was connected with the responsibility of the husband and in about $80 \%$ of all cases, the delivery took place in a hospital.

\section{REFERENCIAS BIBLIOGRAICAS}

1. BERQUO, E. et alli - Levels and variations in fertility revealed by a recent study in São Paulo. [São Paulo, F. H. S. P., 1967] (mimeografado).

2. CABELLO, O. G. - Integralidad del registro de nacimientos $y$ oportunidad de la inscripcion en Chile, 1920-1953. Estadist., Washington, 14(51):302-308, June 1956.

3. COCHRAN, W. G. - Sampling techniques. 2nd ed. New York, Wlley [c963] p. 169.

4. DUNN, H. L. - Uma prova nacional das lacunas do registro de nascimentos nos Estados Unidos. Rev. bras. Estat., 2(6) :225-232, abr./jun. 1941.

5. INSTITUTO BRASILEIRO DE GEOGRAFIA E ESTATISTICA. Conselho Nacional de Estatística - Conjecturas sóbre o nivel da natalidade no Brasil e nas unidades da Federação em 1960. [Rto de Janeiro, 1964] (Pesquisas demográficas, 6).

6. MORAES, N. L. de A. - Estudo sôbre a importancia dos fatôres que podem condicionar a deficiência do registro de nascimentos. Rev. Serv. Saúde públ., Rio de Janeiro, 2(3):743-774, jul. 1949.

7. MORRISON, F. S. \& BLEN, A. L. Metodo de evaluar durante el censo de 1950 la eficacia del registro de nacimientos. Estadist., Washington, $7(25)$ : 571-580, Dec. 1949.

8. OCAMPOS, J. R. \& BENETT, C. G. Prueba de registro de nacimientos en Paraguay. Estadist., Washington, 4(13): 111-116, Mar. 1946.

9. ROSADO, P. - Aspectos do registro civil de nascimientos em uma cidade do interior da Amazônia, 1938-1947. Rev. Serv. Saúde públ., Rio de. Janeiro, 2(3):775-792, jul. 1949.

10. SAADE, M. J. - Verificação estatístíca do grau de deficiencia do registro de nascimentos. Rev. Serv. públ., Rio de Janeiro, 1(2):449-467, jul. 1947.

11. SCORZELLI Jr., A. - Coleta de dados vitais em pequenas localidades. Rev. Serv. Saúde puibl., Rio de Janeiro, 1(2):397-432, jul. 1947.

12. SEMINARIO INTERAMERICANO DE REGISTRO CIVIL, 1.0 , Santiago de Chile, 1954. - Informe final del primer Seminario Interamericano de Registro Civil. Nueva York, Naciones Unidas, 1955. p. 28 (Informes Estadișticos, serie $M, 23$ ).

13. SEMINARIO INTERAMERICANO DE REGISTRO CIVIL, 2.0, Lima, 1964 Aplicación de los acuerdos del primer Seminario de Registro Civil. Nueva York, Naciones Unidas, Consejo Economico y Social, 1964. p. 5 (ST/ECLA) CONF. 19/1.20).

14. SEMINARIO INTERAMERICANO DE REGISTRO CIVIL, 2.0, LIma, $1964-$ Informe sobre el segunido Seminario Interamericano de Registro Civil. Mexico, Comission Economica para America Latina, 1965. (E/CN. 12/704).

15. SHAPIRO, S. \& SCHACHTER, J. Methodology and summary results of the 1950 birth registration test in the United States. Estadist., Washington, 10(37) :688-699, Dec. 1952.

16. SILVA, E. P. C. - Plano de amostragem utilizado no estudo de reproducão humana no Distrito de São Paulo. [São Paulo, F. H. S. P., 1968].

17. SWEE-HOCK, $S$. - A note on the under-registration of births in Malaya during the intercensual period 1947-1957. Popul. Stud., 18(1):35-52, July 1964. 